\title{
Applicable observation of butorphanol in painless colonoscopy examination
}

\author{
Xuefeng $\mathrm{Li}^{1}$, Feng Liang, and Guifeng $\mathrm{Liu}^{2, \mathrm{a}}$ \\ ${ }^{1}$ Department of Anesthesiology, China-Japan Union Hospital, Ji Lin University, Changchun, Jilin, China \\ ${ }^{2}$ Department of Radiology, China-Japan Union Hospital, Ji Lin University, Changchun, Jilin, China
}

\begin{abstract}
Objective:To summarize the clinical effect of butorphanol compound propofol in painless colonoscopy examination and its feasibility.Methods:100 colonoscopy examination patients (56 males and 44 females aged from 19 to 60 years old) registered between August, 2016 and September, 2016 in the endoscopy center of our hospital were randomly selected. ASA classification is I or II level. Their body weight ranged from $55 \mathrm{~kg}$ to $75 \mathrm{~kg}$. They were randomly divided into two groups and each group included 50 cases. All patients went through conventional ambrosia and liquid fasting for 8 hours before the anesthesia and they drank magnesium sulfate liquid of $2500 \mathrm{ml}$ to clean their gastrointestinal tracts.After patients entered the operating room, their veins of upper limb were opened so as to monitor their HR, MAP and $\mathrm{SPO}_{2}$. After that, butorphanol of $20 \mu \mathrm{g} / \mathrm{kg}$ was injected to patients of the experimental group while normal saline of the same amount of was injected to patients of the contrast group. After 60 seconds, propofol of $1 \sim 2 \mathrm{mg} / \mathrm{kg}$ was injected to both groups by the way of intravenous injection. The enteroscopy examination was started after patients had no eyelash reflection. Besides, actual application dose of propofol was adjusted according to clinical indications of patients and the adjusting frequency each time was controlled between 30 milligrams and 50 milligrams until the completion of the examination. SPSS 22.0 statistical software was used to analyze and handle research data of this group. Results:Anesthesia effect: The difference of inter-group comparison showed no statistical significance $(\mathrm{P}>0.05)$. The intra-group comparison and the inter-group comparison show that the difference in terms of changes of HR, MAP and $\mathrm{SpO}_{2}$ of patients in two groups before and after the anesthesia had no statistical significance $(\mathrm{P}>0.05)$. The awakening time, VAS score, postoperative vomiting times and the occurrence rate of respiratory depression of the observation group were all significantly lower than those of the contrast group $(\mathrm{P}<0.05)$.
\end{abstract}

\section{Introduction}

In order to summarize the clinical effect of butorphanol compound propofol in painless colonoscopy examination and its feasibility, this research randomly selected 100 colonoscopy examination patients from the endoscopy center of our hospital and divided them into two groups. During the examination link, the contrast group only uses the propofol while the experimental group uses butorphanol compound propofol. Based on the observation results of the anesthesia effectiveness of two groups of patients, this paper studied the anesthesia analgesia effect of butorphanol in its application in painless colonoscopy. Specific contents are as follows

\footnotetext{
a Corresponding author: 28186282@qq.com
} 


\section{Materials and Methods}

This study was approved by Ethics Committee of our hospital. In addition, our hospital has signed the informed consent form with all patients. 100 colonoscopy examination patients (56 males and 44 females aged from 19 to 60 years old) registered between August, 2016 and September, 2016 in the endoscopy center of our hospital were randomly selected. They all belonged to Level I to Level II of ASA and had no obvious heart and lung diseases. Their body weight ranged from $55 \mathrm{~kg}$ to $75 \mathrm{~kg}$. They were randomly divided into two groups and each group included 50 cases. The gender, age and body weight differences of patients in both groups had no statistical significance. All patients went through conventional ambrosia and liquid fasting for 8 hours before the anesthesia and they drank magnesium sulfate liquid of $2500 \mathrm{ml}$ to clean their gastrointestinal tracts. No one had the experience of preanesthetic medication. After patients entered the operating room, their veins of upper limb were opened so as to monitor their HR, MAP and $\mathrm{SPO}_{2}$. Then, they absorbed oxygen $(3 \mathrm{~L} / \mathrm{min})$. After that, butorphanol of $20 \mu \mathrm{g} / \mathrm{kg}$ was injected to patients of the experimental group while normal saline of the same amount of was injected to patients of the contrast group. After 60 seconds, propofol of 1 2 $\mathrm{mg} / \mathrm{kg}$ was injected to both groups by the way of intravenous injection. The enteroscopy examination was started after patients had no eyelash reflection. Besides, actual application dose of propofol was adjusted according to clinical indications of patients and the adjusting frequency each time was controlled between 30 milligrams and 50 milligrams until the completion of the examination. SPSS 22.0 statistical software was used to analyze and handle research data of this group. The measurement data were shown by $(\mathrm{x} \pm \mathrm{s})$ and inter-group comparison adopted the way of t-test. In addition, the counting data were expressed by the case number (n) and the comparison of intra-group rate (\%) used the way of $\mathrm{X} 2$-inspection. $\mathrm{P}<0.05$ means that the difference was statistically significant.

\section{Results}

Anesthesia effect: There were 48 excellent cases and two good cases in the observation group in terms of the anesthesia effect and 45 excellent cases, 4 good cases and one bad case in the contrast group. The excellence rate of the observation group was $100.00 \%$, which was slightly higher than that of the contrast group (98\%). The difference of inter-group comparison showed no statistical significance $(\mathrm{P}>0.05)$.

Vital signs: The intra-group comparison and the inter-group comparison show that the difference in terms of changes of HR, MAP and $\mathrm{SpO}_{2}$ of patients in two groups before and after the anesthesia had no statistical significance $(\mathrm{P}>0.05)$. Please refer to Table 1 .

Table 1. Change of vital signs of two groups of patients before and after the anesthesia $(\mathrm{x} \pm \mathrm{s})$

\begin{tabular}{ccccc}
\hline Time & Groups & HR (Times $/ \mathrm{min})$ & $\mathrm{MAP}(\mathrm{mmHg})$ & $\mathrm{SpO}_{2}(\%)$ \\
\hline Before anesthesia & Observation group & $80.23 \pm 8.75$ & $83.43 \pm 9.67$ & $98.23 \pm 1.10$ \\
& Contrast group & $81.37 \pm 8.67$ & $82.79 \pm 9.21$ & $98.11 \pm 1.03$ \\
$\begin{array}{c}\text { After the } \\
\text { medicine }\end{array}$ & Observation group & $74.47 \pm 7.55$ & $72.18 \pm 7.35$ & $96.37 \pm 2.26$ \\
$\begin{array}{c}\text { is used for 1 min } \\
\text { After the }\end{array}$ & Contrast group & $5.88 \pm 7.47$ & $71 . .26 \pm 7.21$ & $96.06 \pm 2.35$ \\
inspection & Observation group & $78.96 \pm 7.98$ & $81.57 \pm 9.46$ & $97.53 \pm 1.52$ \\
& Contrast group & $79.87 \pm 8.06$ & $80.78 \pm 9.24$ & $97.41 \pm 1.50$ \\
\hline
\end{tabular}

Awakening time and adverse reaction: Patients of the observation group needed less awakening time than those of the contrast group. Comparison among groups showed no statistical significance $(\mathrm{P}>0.05)$. Besides, the awakening time, VAS score, postoperative vomiting times and the occurrence rate of respiratory depression of the observation group were all significantly lower than those of the contrast group $(\mathrm{P}<0.05)$. Please refer to Table 2 . 
Table 2 Comparison of awakening time and adverse reaction [ $(\mathrm{x} \pm \mathrm{s}), \mathrm{n}(\%)$ ]

\begin{tabular}{|c|c|c|c|}
\hline Group & $\begin{array}{l}\text { Awakening time } \\
\text { (min) }\end{array}$ & VAS Score & $\begin{array}{c}\text { Postoperative } \\
\text { vomiting (times) }\end{array}$ \\
\hline
\end{tabular}
Observation group
$2.68 \pm 1.02$
$1.06 \pm 0.32$
$1.16 \pm 0.30$
$1(2 \%)$

Contrast group

$3.06 \pm 1.39$

$2.10 \pm 0.56$

$2.16 \pm 0.58$

$7(14 \%)$

\section{Discussion}

Propofol has many advantages, such as no accumulation, fast effect and relatively short lasting period $^{[1]}$, and it is widely used in the field of painless gastroscopy. Even so, propofol is very limited in its analgesic effect because its anesthesia procedures can only be completed with the aid of other drugs, of which, butorphanol is the most frequently used kind. Butorphanol is a kind of mixed opioid receptor agonists-opioid receptor-antagonists and its receptor owns distinctiveness of features ${ }^{[2]}$. Thus, it can control the situation of respiratory depression and avoid the appearance of unsatisfactory situation of patients' gastrointestinal tracts. Besides, patients are not addicted to it and its drug efficacy can last relatively longer, thus, it is popular in clinical practice ${ }^{[3]}$.

According to current clinical practices, propofol shall be used together with other analgesic drugs or local anesthetics for the conduction of anesthesia because this can not only improve the analgesic effect, but also reduce the adverse reactions caused by anesthesia medicines. This study uses tartaric acid butorphanol compound propofol to the anesthesia of painless colonoscopy examination ${ }^{[4]}$. The results show that its anesthesia effect is slightly higher than that of the contrast group and the pain degree (VAS) caused by it is significantly lower than that of the contrast group. This indicates that the tartaric acid butorphanol tartrate compound propofol has good anesthesia effect and it can effectively reduce the pain degree of enteroscopy examination patients. Butorphanol is a kind of opioid receptor agonists-opioid receptor excited-antagonists and it exerts analgesic effect by acting on k-receptors. Literature ${ }^{[5]}$ points out that the analgesia intensity of butorphanol is five to eight times of that of morphine and it can promise quick returns because its analgesic effect can be played within one minute after the intravenous injection. The simultaneous usage of propofol and butorphanol can exert very good anesthetic effect and effectively suppress the pain of the injection site, which will eventually promote the smooth conduction of painless colonoscopy examination. Anesthesiologists always focus on the vital signs of patients and the security of anesthesia process. In this study, inter-group comparison and intra-group comparison of two groups of patients show that changes of $\mathrm{HR}, \mathrm{MAP}$ and $\mathrm{SpO} 2$ have no obvious difference before the anesthesia, within one minute after the medicine is used and after the anesthesia. This presents that butorphanol compound propofol anesthesia do not affect vital signs of patients. Butorphanol, which is a kind of opioid receptor antagonists, exerts no respiratory depression, thus, it can effectively maintain the stability of respiration and circulation in the anesthesia process. In addition, butorphanol has weak antagonism to human $\mu$ opioid receptor and it can reduce adverse reactions of gastrointestinal tracts and the occurrence rate of nausea and vomiting after the anesthesia ${ }^{[6]}$. It can reach its peak value within four to five minutes after the intravenous injection and patients can regain consciousness fast. This research shows that patients in the observation group only need a short awakening time and the occurrence rate of their postoperative vomiting times and respiratory depression effect are all significantly lower than those of the contrast group. Domestic and foreign scholars also had similar literature reports before, showing that it is safe to use butorphanol to the anesthesia of painless colonoscopy examination.

In conclusion, butorphanol compound anesthesia is helpful to ease the pain degree, reduce the 
adverse reaction, improve the anesthesia quality and promote the smooth operation of painless colonoscopy examination. Limitations of this study lie in that anesthesia time points are not subdivided and its functional mechanisms are not sufficiently analyzed. Thus, more in-depth researches still remain to be done in the future.

\section{Conclusion}

Butorphanol compound anesthesia is helpful to ease the pain degree, reduce the adverse reaction, improve the anesthesia quality and promote the smooth operation of painless colonoscopy examination.

\section{References}

1. Yingchao Xie, Jingxian Seng, Xichao Zhu, ZhiHeng Ke, Yingsheng Bo, Yingting Liang. Different dose of miss bhutto's brown composite application of propofol in painless tubal ligation [J].Practical medical journal 201329(10):1665-1667(in Chinese).

2. Bailey PL,Egan TD,Stanley TH.Intravenous opioid anesthetics[M].Miller RD.Anesia.5th ed.Philadelphia:Churchill Livingstone,2000:273-376.

3. Arora V,Bajwa SJ,Kaur S.Comparative evaluation of recovery characteristics of fentanyl and butorphanol when used as supplement to propofol anaesthesia[J].Int J Appl Basic Med Res,2012,2(2):97-101.

4. Gupta A,Kaur S,Attri JP, et al.Comparative evaluation of ketamine - propofol,fentanyl - propofol and butorphanol-propofol on haemodynamics and laryngeal mask airway insertion conditions[J].J Anaesthesiol Clin Pharmacol,2011,27(1):74-78.

5. Kaur J,Srilata M,Padmaja D,et al.Dose sparing of induction dose of propofol by fentanyl and butorphanol: A comparison based on entropy analysis[J].Saudi J Anaesth,2013,7(2):128-133.

6. Ishizuka T,Itami T,Tamura J,et al.Anesthetic and cardiorespiratory effects of propofol,medetomidine,lidocaine and butorphanol total intravenous anesthesia in horses[J].J Vet Med Sci,2013,75(2):165-172. 